\title{
Trayectoria Escolar y Ranking: valoraciones y estrategias institucionales en el nuevo escenario de selección universitaria
}

\author{
School career and Ranking: valuations and institutional strategies \\ in the new scenario of university selection
}

\author{
Carlos Rodríguez Garcés, ${ }^{a}$ Geraldo Padilla Fuentes ${ }^{b}$ \\ ${ }^{a}$ Departamento de Ciencias Sociales, Universidad del Bío-Bío \\ Director del Centro de Investigación CIDCIE \\ Telf.: (56) 42 2463616. Correo electrónico: carlosro@ubiobio.cl \\ ${ }^{\mathrm{b}}$ Centro de Investigación CIDCIE, Universidad del Bío-Bío \\ Telf.: (56) 42 2463616. Correo electrónico: gpadilla@alumnos.ubiobio.cl
}

\begin{abstract}
RESUMEN
El acceso a la educación universitaria selectiva en Chile ordena a los postulantes de acuerdo a un puntaje que pondera los factores de Trayectoria Escolar y pruebas PSU. En 2013, la inclusión del Ranking de Notas ocasionó una reestructuración en la ponderación de los factores de selección, redefiniendo las posiciones institucionales al respecto. Con bases de datos oficiales y procedimientos estadísticos ad hoc, se describe el comportamiento de los 1423 programas de pre-grado ofertados por las 33 universidades integrantes del Sistema Único de Admisión en 2014. Se observa que el componente PSU pierde hegemonía comparado con la Trayectoria Escolar como factor de ponderación, resignificándose especialmente el Ranking. Esta es una tendencia generalizada, pero de comportamiento no homogéneo. Posiciones institucionales escindidas en la valoración del desempeño escolar, a partir del nivel de selectividad tradicional del programa, evidencian estrategias diferenciadas de reclutamiento en una gradiente de adhesión a políticas pro inclusión y defensa de intereses institucionales.
\end{abstract}

Palabras clave: Ranking de notas, Trayectoria Escolar, selección universitaria, inclusión educativa, educación superior.

\section{ABSTRACT}

In Chile, the access to selective university education is developed according to the average score obtained by applicants at school plus the score obtained in the PSU test. In 2013 the incorporation of the School Grades Ranking generated a reframing in the weighting of selection factors, redefining institutional position towards it. Based on official data and adhoc statistical procedures, the behavior of 1423 undergraduate programs offered by the 33 universities which are part of the 2014 Admission System is described. It is possible to observe that PSU test loses its main position when compared to school's result as the weighting factor, directly affecting the Ranking. This is the predominant trend but with a non-hegemonic behavior. Institutional position framed upon the school performance proves different strategies to attract applicants from an approach which encourages inclusion and the defense of institutional interests.

Key words: Ranking of Score, school career, university selection, inclusive education, higher education. 


\section{INTRODUCCIÓN}

En Chile, el sistema de educación superior está compuesto por centros de formación técnica, institutos profesionales y universidades, que al término de los procesos formativos que imparten conceden títulos técnicos de nivel superior, títulos profesionales y grados académicos respectivamente (Sverdlick, Ferrari y Jaimovich, 2005). El ingreso a las universidades tiene un comportamiento dual. Por una parte, se tiene un conglomerado de instituciones selectivas que definen un conjunto de reglas y procedimientos compartidos por los cuales se rigen y que están expresados en el Sistema Único de Admisión. Por otro, un conjunto de instituciones de naturaleza privada, autónomas en la definición de sus procesos de admisión, cuya oferta educativa cubre cerca del $65 \%$ de la matrícula de educación superior (Espinoza, 2015).

Actualmente, 33 universidades adhieren al Sistema Único de Admisión (SUA), correspondiente a las 25 que integran el Consejo de Rectores de las Universidades Chilenas $(\mathrm{CRUCH})$, además de las 8 universidades privadas, y que en su conjunto ofertan un total de 1423 programas formativos. Este sistema contempla el rendimiento de un conjunto de pruebas de selección que realizan los egresados de la enseñanza media. Test que en la actualidad, en razón de las políticas de inclusión desarrolladas por el Ministerio de Educación a partir de 2007, alcanza coberturas casi universales de realización para la promoción del año. Son los puntajes alcanzados en estos test y las notas de enseñanza media los factores históricos de ponderación que contemplaba el sistema (Contreras, Corbalán y Redondo, 2007), cuya valoración definía con relativa autonomía las universidades para el ingreso a sus programas.

Esta modalidad de ingreso se mantuvo sin grandes cambios por cerca de 60 años, situación que el 2013 marca un punto de inflexión en esta tendencia al contemplarse la inclusión del Ranking de Notas como factor de ponderación complementario de las NEM en el reconocimiento de la Trayectoria Escolar y los atributos intrínsecos de los postulantes, independientemente de sus condiciones de origen (Meneses y Blanco, 2006; Meneses, Parra y Zenteno, 2005). El proceso de postulación y selección a la educación superior transita por una nueva etapa de discusión y análisis en busca de cumplir con criterios de igualdad y equidad para reducir la segregación social y escolar, al igual que otros países de América Latina (Bellei, 2013; Chiroleu, 2009).

Cuenta de lo anterior han dado las diferentes universidades nacionales vinculadas al SUA, que desde 2013, además de ponderar la puntuación alcanzada en algunas pruebas estandarizadas (Matemáticas, Lenguaje, Ciencias Sociales y Naturales) y el promedio de Notas de la Enzeñanza Media (NEM) como predictores del futuro nivel de logro, comienzan a ponderar también el Ranking de Notas, entendido como la posición relativa que ocupa el alumno en su establecimiento de egreso en relación a la posición histórica que considera las tres generaciones anteriores (Larroucau, Ríos y Mizala, 2013). Estos dos factores (NEM y Ranking) vienen a fortalecer la valoración que se da a la Trayectoria Escolar en comparación a la dimensión PSU (Matemáticas, Lenguaje, Ciencias Sociales y Naturales), la que tiene por finalidad medir el manejo que los alumnos tienen de los contenidos curriculares secundarios impartidos por los colegios bajo dirección del Ministerio de Educación.

El Ranking de notas como factor de ponderación es una herramienta utilizada desde hace años por algunos países para mejorar el cumplimiento de objetivos orientados a la integración social y cultural (Cliffordson y Askling, 2006), temáticas que en Chile se han 
vuelto necesarias de mejorar y transparentar mediante algunas modificaciones al proceso de transición de la educación secundaria a la superior (Díaz, Himmel y Maltes, 1990).

La evidencia plantea un conjunto de beneficios que conllevaría el reconocimiento de las habilidades desarrolladas y esfuerzo desplegado en los procesos de educación secundaria para la realización de estudios superiores, más allá de las competencias o conocimientos expresados en una prueba estandarizada aplicada en condiciones desiguales de preparación (Medina, Aguirre y Luengo, 2014). Variadas investigaciones, tanto nacionales como internacionales, reconocen que un buen predictor del rendimiento académico superior es la posición relativa que el estudiante alcanza en su establecimiento de egreso a partir de características como: la motivación, la disposición al estudio o el aprovechamiento de los contenidos, aspectos que no captarían las pruebas estandarizadas (Contreras, Gallegos y Meneses, 2009; Gallegos y Meneses, 2007; Geiser y Studley, 2002; Gil, 2006; Gil y Canto, 2012; Gil, Paredes y Sánchez, 2013; Meneses y Toro, 2012; Román, 2012). Además, un mayor reconocimiento a la Trayectoria Escolar, y en especial al Ranking, aportaría a políticas de inclusión disminuyendo la normativización de la población educativa que accede a determinados programas selectivos y que comparten homogéneas características sociofamiliares (Valdivieso, Antivilo y Barrios, 2006).

El proceso de selección universitaria 2014 fue el primero en permitir a las universidades ponderar con relativa autonomía el más reciente de los factores de selección, en comparación al año anterior en que Ranking tuvo una ponderación fija de 10\%. Las restricciones adicionales regulan que las Pruebas de Selección Universitaria (PSU) deben ser ponderadas en un mínimo de $10 \%$ y máximo $40 \%$, considerando las dos pruebas obligatorias y la optativa que varía de una carrera a otra. La suma de estas ponderaciones no debe ser inferior a 50\%. En la otra dimensión, Ranking y NEM tienen un mínimo de $10 \%$ y un máximo de $40 \%$ cada una, la suma de ambas no puede exceder el $50 \%$ por las restricciones a la dimensión PSU (Larroucau, 2014).

Esta facultad para determinar el valor de los requerimientos de ingreso a las carreras es una manifestación del nivel de adhesión institucional, en la forma y en el fondo, a la intencionalidad de la política social que motivó la modificación del sistema de admisión. Este artículo se basa en el supuesto que la oferta educativa institucional no tendría un comportamiento homogéneo a este respecto, transitando en un cuadrante que cruza selectividad e inclusión, conforme otorguen más o menos valoración al componente PSU, o, en su defecto, a la Trayectoria Escolar. Análisis de la toma de posición de la oferta institucional que permitirá vislumbrar las lógicas subyacentes de reclutamiento de la demanda educativa a matricular en sus programas.

\section{METODOLOGÍA}

\subsection{DISEÑO}

La investigación hace uso de una base de datos ad hoc, construida a partir de los informes de ponderaciones de la oferta educativa para los procesos de admisión 2012, 2013 y 2014, los cuales son proporcionados por el Departamento de Evaluación, Medición y Registro Educacional (DEMRE), que tiene por cargo administrar el Sistema Único de Admisión a la universidad vía PSU, cuyos informes son publicados anualmente en los 
diarios oficiales de este organismo. Esta información es complementada a partir de los datos recogidos en otras bases o informes de carácter oficial para el territorio nacional, como por ejemplo la base "Índices" del Consejo Nacional de Educación, lo que posibilita construir nuevos indicadores para la unidad de observación "programas".

Con esta base de datos de fuente secundaria, oficial y de acceso público, se aborda el análisis del comportamiento que presentan los distintos factores de ponderación utilizados en el proceso de admisión universitaria, en específico, los componentes de rendimiento PSU (puntajes en las pruebas estandarizadas de Matemáticas y Lenguaje) y los de Trayectoria Escolar (NEM y Ranking). Análisis que tiene por finalidad determinar los comportamientos diferenciales que evidencia la oferta educativa sobre la base de la valoración de los factores de ponderación, procurando develar las estrategias de reclutamiento y lógicas de segmentación que establecen instituciones y programas en sus diferenciales comportamientos.

\subsection{MUESTRA}

La muestra de estudios estuvo constituida por los 1260, 1324 y 1423 programas de pregrado que fueron ofertados en los procesos de admisión universitaria 2012, 2013 y 2014 respectivamente, pertenecientes a las 25 universidades públicas y 8 privadas que forman parte del CRUCH. Estas universidades tienen la autonomía administrativa de fijar sus planes y programas de estudio, los que se vinculan al Sistema Único de Admisión a cargo del Departamento de Evaluación, Medición y Registro de la Educación.

\subsection{INSTRUMENTOS Y PROCEDIMIENTO}

Se recoge para cada año y programa ofertado las valoraciones, en términos de ponderación asignada, de cada uno de los componentes del sistema de selección universitario: Trayectoria Escolar y dimensión PSU.

La dimensión PSU considera en términos porcentuales el peso establecido por cada uno de programas a las pruebas estandarizadas de Lenguaje, Matemáticas y Ciencias. Test que expresan el nivel de dominio del currículum de enseñanza media que tiene el alumno y que en teoría lo haría apto para el desarrollo de trayectorias educativas más exigentes. Estos puntajes son normalizados en una escala común de 500 puntos promedio y desviación estándar de 100 puntos, los que se sitúan en un gradiente que transita de los 150 hasta los 850 puntos.

La dimensión Trayectoria Escolar está conformada por las ponderaciones asignadas a los factores NEM y Ranking. Las NEM, en cuanto a calificación, son la expresión del rendimiento escolar y aprovechamiento del currículum desarrollado por el estudiante en los cuatro años que corresponden a la enseñanza media en Chile. Estas calificaciones son convertidas, dependiendo de la rama de enseñanza, a puntuación estándar PSU para hacerlas homologables al resto de factores de ponderación. El Ranking de notas se refiere a la posición relativa que ocupa el estudiante en su establecimiento de egreso al final de su recorrido escolar por la enseñanza media. Este factor busca medir, complementario a las NEM, el desempeño académico a partir del contexto educativo donde desarrollan las experiencias de enseñanza-aprendizaje. La conversión a puntaje de esta conceptualización toma como referencia el rendimiento escolar de los alumnos egresados en las tres generaciones egresadas anteriormente del colegio, esto con el fin de evitar la proliferación de lógicas competitivas y de inflación de notas dentro de los establecimientos. 
Para fines de análisis, se construyó una variable de segmentación en referencia al nivel de selectividad que evidenciaba la unidad de observación o programa educativo. Para ello se consideró la proporción de estudiantes que en años anteriores teniendo un determinado puntaje de corte en las pruebas PSU Matemáticas y Lenguaje adherían a dicho programa. Se distinguen así tres segmentos o grupos: alta selectividad, más del 50\% de su matrícula histórica tiene puntajes por sobre los 650 puntos PSU promedio; mediana selectividad, definido como aquellos programas donde más del $50 \%$ de su matrícula tiene entre 550 y 650 puntos PSU promedio; por último, los de baja selectividad, donde la mitad o más de su alumnado no supera la barrera de los 550 puntos PSU ponderados.

\section{RESULTADOS Y DISCUSIONES}

El ingreso a la educación superior de carácter selectiva se realiza mediante un proceso de admisión que ordena a los postulantes a partir de un puntaje en cuyo cálculo intervienen dos componentes generales de ponderación. El primero de ellos, "Resultados PSU", recoge el rendimiento expresado en puntaje alcanzado en las pruebas ad hoc de selección universitaria. El segundo, "Trayectoria Escolar", pretende valorar el nivel de logro y esfuerzo desplegado por el estudiante durante su desarrollo escolar pre universitario a través de la ponderación de las notas de la enseñanza media y la posición relativa o ranking del estudiante en su respectivo establecimiento. Teniendo las universidades y programas de la oferta educativa disponible la autonomía relativa de asignar, dentro de determinados límites, diferenciadas ponderaciones a los dimensiones "Resultados PSU" y "Trayectoria Escolar", la valoración que ellas otorgan a dichos componentes del proceso de admisión universitaria sería una expresión de mayor o menor respaldo o sintonía con las modificaciones realizadas recientemente al mecanismo de selección. En síntesis, establecerían un posicionamiento frente a la capacidad predictiva que tendría la valoración de la Trayectoria Escolar en cuanto a la selección de buenos alumnos, así como el grado de confianza que le conceden a los tradicionales procesos de selección.

Gráfico 1. Valoración de la dimensiones Trayectoria Escolar y PSU en los procesos de selección universitaria 2012 al 2014 según categorías de segmentación

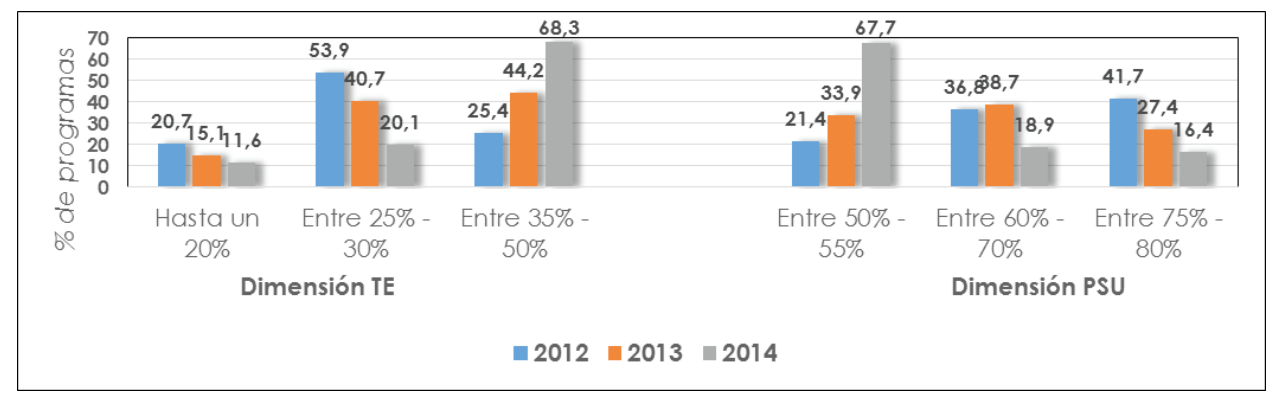

Fuente: DEMRE (2013). 
El Gráfico 1 muestra la valoración que las universidades han otorgado a los factores de ponderación los últimos tres años según Trayectoria Escolar (NEM Y Ranking) y PSU (Lenguaje y Matemáticas). Según disposiciones reglamentarias vigentes que rigen los procesos de admisión, la ponderación mínima posible para Trayectoria Escolar es de un $20 \%$ repartido igualitariamente entre complementarios, alcanzando un máximo de 50\%. Del abanico de posibles ponderaciones, optar por darle a la Trayectoria Escolar una valoración del $20 \%$ ha sido, especialmente en los últimos dos años, la opción menos acogida. Con independencia del año de estudio, entre un 20,7\% y un 11,6\% de la oferta educativa existente asigna la ponderación mínima reglamentaria a este componente. En la actualidad, tan solo 1 de cada 10 programas ofertados están en los mínimos legales, observándose por parte de las instituciones una tendencia a asignar una menor ponderación al factor PSU.

Durante el 2012, 1 de cada 2 programas $(53,9 \%)$ asignaba a la Trayectoria Escolar una ponderación de entre el $25 \%$ y el $30 \%$, valoraciones superiores eran asignadas al $25 \%$ de la oferta educativa. Esta ponderación intermedia (entre 25\% y $30 \%$ ) también ha disminuido en los programas ofertados en cada año. En el 2014 tan solo el 20,1\% de los programas establecen esta ponderación, lo que representa una disminución del $64 \%$ respecto de la existente en el 2012. En consecuencia, se ha dado paso a un fenómeno de creciente alza en la cantidad de programas que valoran como requisito de ingreso a las universidades la Trayectoria Escolar. Prueba de aquello es que 7 de cada 10 programas (68,3\%) en 2014 exhiben una valoración entre NEM y Ranking superior al 35\%.

Como corolario de lo anterior se desprende complementariamente una pérdida de hegemonía en términos de importancia porcentual de las pruebas de selección mismas. Al año 2014, el 67,7\% de la oferta educativa de educación superior no otorga al componente PSU ponderaciones superiores al 55\%. Asimismo, la cantidad de carreras que otorga una alta valoración a la dimensión PSU, esto entre un $75 \%$ y $80 \%$, ha disminuido a más de la mitad de lo que acontecía en 2012 (16,4\% frente a 41,7\%). Cabe hacer presente aquí dos hechos esenciales. Primero, que una mayor depreciación del componente PSU choca con barreras administrativas que establecen que los mínimos legales vigentes a la fecha son de un 50\%. Segundo, que la decisión de asignar esta baja ponderación al componente PSU era seguida en el 2012 por tan solo el 21,4\% de los programas, es decir, tres veces menor a la existente en la actualidad.

Cuadro 1. Valoración de la Trayectoria Escolar según tipo de Universidad y año de proceso

\begin{tabular}{|l|c|c|c|c|c|c|}
\hline Tipo de institución universitaria & 2012 & 2013 & 2014 & $\begin{array}{c}\text { VTE 2012 } \\
\text { al 2013 }\end{array}$ & $\begin{array}{c}\text { VTE 2013 } \\
\text { al 2014 }\end{array}$ & $\begin{array}{c}\text { VTE 2012 } \\
\text { al 2014 }\end{array}$ \\
\hline - Pública Tradicional & 30,1 & 35,2 & 42,1 &, $17(, 19)$ &, $23(, 27)$ &, $42(, 32)$ \\
\hline - Privada & 26,2 & 26,9 & 28,0 &, $05(, 16)$ &, $06(, 30)$ &, $08(, 30)$ \\
\hline Totales & 29,2 & 33,2 & 38,8 &, $14(, 19)$ &, $19(, 29)$ &, $34(, 35)$ \\
\hline
\end{tabular}


En el Cuadro 1 aparecen las ponderaciones promedio asignadas al componente Trayectoria Escolar por parte de las instituciones educativas públicas y privadas en los tres años que abarca el estudio. Históricamente, la selectividad se ha traducido operacionalmente en una alta ponderación del componente PSU, en detrimento de la valoración de la Trayectoria Escolar. Hasta el 2012, no obstante las diferencias al interior del sistema, alrededor de 7 de cada 10 puntos del puntaje ponderado con que postulaban los alumnos a los diversos programas provenían del componente estandarizado. En dicho año, de los 1260 programas existentes, un 74,6\% asignaba a la Trayectoria Escolar una valoración igual o inferior a 30 puntos ponderados, constatándose además un diferencial comportamiento según tipo de institución, siendo las de naturaleza Privada quienes menor ponderación otorgaban a este factor, el 85,6\% no superaba la barrera de los 30 puntos ponderados asignados en comparación al $71,2 \%$ de su contraparte pública (ver Gráfico 1). Durante los años sucesivos se constata un parcelado pero significativo aumento en el reconocimiento del rendimiento escolar previo expresado en NEM y Ranking, situándose en el año 2014 en el 38,8\% promedio a nivel general, lo que implica un aumento del orden del $33 \%$ del existente en el 2012.

En consecuencia, no tan solo se deprecia la relevancia y excesiva hegemonía del componente PSU en la asignación de puntaje; sino que, además, aumentan considerablemente las brechas entre públicas y privadas a este respecto. El sistema de admisión se segmenta sobre la base de la valoración que hacen unas y otras a la Trayectoria Escolar; en 2014, de los estudiantes que postularon a universidades públicas, el ranking y el NEM equivalieron en promedio al $42,1 \%$ de su puntaje ponderado, mientras que aquellos que apostaron por alguna universidad privada aprovecharon, en promedio, un $28 \%$ su rendimiento escolar previo para aumentar su puntaje y postular, situación favorable para algunos, tomando en consideración que de haber participado del proceso de selección 2012 en el mismo sector hubieran perdido cerca de un $2 \%$ de su Trayectoria Escolar. Esto, aunque leve, muestra cambios en ambos sectores educativos.

Cuadro 2. Valoración de los factores de ponderación en el proceso de selección universitaria 2014 y sus variaciones respecto al 2013

\begin{tabular}{|l|c|c|c|c|c|c|}
\hline \multicolumn{2}{|c|}{} & \multicolumn{2}{c|}{ Componente TE } & & \multicolumn{2}{c|}{ Componente PSU } \\
\hline Tipo de Institución & VTE & NEM & RANK & VPSU & MATE & LENG \\
\hline Pública Tradicional & $42.1(22,8)$ & $16.3(-29,6)$ & $28.8(156,7)$ & $57.9(-9,6)$ & $25.8(-8,5)$ & $19.4(-9,1)$ \\
\hline Privada & $28.0(5,7)$ & $14(-3,17)$ & $14(40)$ & $72(-1)$ & $32.0(-4,5)$ & $25.3(-3,1)$ \\
\hline Total & $38.0(18,9)$ & $15.8(-25,9)$ & $23(128,6)$ & $61.2(-7,6)$ & $27.3(-7,5)$ & $20.8(-7,6)$ \\
\hline
\end{tabular}

Nota: VTE=Valoración Trayectoria Escolar; NEM= Notas de enseñanza media; RANK=ranking; VPSU= Valoración PSU; MATE= Prueba Matemáticas; LENG= Prueba Lenguaje. 
El Cuadro 2 muestra las variaciones porcentuales de los factores de ponderación en el proceso de selección 2014 respecto al 2013, controlando por tipo de institución. A nivel general y con independencia del tipo de institución y programa ofertado, la Trayectoria Escolar se valoriza en promedio un $18,9 \%$ respecto de las ponderaciones asignadas el 2013, ello, como es lógico, en detrimento del componente PSU, el cual pierde un 7,6\% promedio de su ponderación asignada a igual año, con escasa diferencia respecto de tipo de prueba. Actualmente, 2 de cada 3 puntos en promedio que obtiene un alumno al postular a una universidad que participa del proceso de admisión provienen del componente Trayectoria Escolar. A nivel desagregado, no obstante, el factor que adquiere mayor relevancia en la Trayectoria Escolar es el Ranking, registrando un crecimiento en su valoración de un 128,6\%, y se sitúa en el orden de los 23 puntos porcentuales como factor. Las NEM, por su parte, pierden un cuarto de su ponderación promedio existente al 2013 (25,9\%), y en promedio pondera un 15,8.

Asimismo, se observan diferenciales comportamientos según la naturaleza de la oferta educativa. Las instituciones públicas tienden en promedio, y no obstante los matices, a favorecer la Trayectoria Escolar, en especial el ranking, significativamente más que su contraparte privada. En estas universidades tradicionales, de cada 100 puntos 42 son obtenidos por el componente Trayectoria Escolar, y de estos, 29 provienen del ranking, el cual evidencia un cambio en su valoración cercano al 157\% respecto del que tenía el 2013. En el sector privado, por su parte, es el componente PSU el de mayor ponderación. 72 de cada 100 puntos se derivan de este concepto, y si bien la Trayectoria Escolar y el ranking se valorizan, lo hacen en una proporción significativamente menor que la constatada en las instituciones consideradas tradicionales.

En consecuencia, los cambios en la oferta educativa no tan solo se manifiestan en una mayor valoración del componente Trayectoria Escolar, especialmente evidente en el sector público, sino que básicamente en una resignificación del Ranking. Se constata además una tendencia a reasignar ponderaciones desde el NEM hacía el Ranking, más que a extraerla únicamente del componente PSU. En otras palabras, puestos a elegir, las instituciones educativas le concederían un mayor nivel de confianza en el proceso de selección a la posición relativa que ocupa el alumno dentro de su establecimiento, más que al nivel de logro expresado en una calificación, que muchas veces resulta artificiosamente abultada fruto de diferenciados niveles de exigencia y políticas evaluativas definidas a partir del contexto socioeducativo o perfil del alumnado.

Las ponderaciones a los factores de selección modelan el perfil de la cuota estudiantil que accede a las ofertas de pregrado, condicionando de paso las posibilidades de ingreso que tienen los postulantes. De tal forma, el acceso no está configurado exclusivamente por el rendimiento obtenido por el estudiante en cada uno de los componentes PSU y Trayectoria Escolar, sino que además en las valoraciones que las instituciones educativas hacen de ellos, siendo finalmente las universidades las que eligen. Selección e inclusión son términos que tienden a contraponerse en el proyecto educativo tradicional. Un menor nivel de selectividad democratiza el acceso a través del aumento de la matrícula, permitiendo la inclusión de una masa de estudiantes de condiciones socioeducativas muy variadas que antes se veían impedidos de ingreso por no traspasar la barrera de admisibilidad o puntaje de corte definido. Pero dentro de sus bemoles, una menor selectividad disminuiría los niveles de eficiencia interna, alteraría los costos de oportunidad disminuyendo los niveles de persistencia en la opción educativa elegida, así como también haría más compleja la formación profesional por un déficit de competencias educativas en el alumnado. 
En consecuencia, las universidades definirían sus estrategias de reclutamiento en una gradiente de selectividad e inclusión. En la búsqueda de cumplir con criterios de equidad y calidad en la selección e integración a la educación superior, las universidades juegan un rol validador de la política social y de sus estrategias depende que las modificaciones cumplan con los objetivos propuestos. Las instituciones y programas más selectivos exhibirán un comportamiento diferenciado en la valoración de los factores de ponderación, considerando a la PSU un eficiente mecanismo de selección. Estas estimarían que la ponderación PSU da mayores garantías de reclutar a un contingente de postulantes de adecuadas condiciones de educabilidad, quienes desarrollarían trayectorias académicas de exigencia sin mayores sobresaltos que resten eficiencia al sistema. Más aún, si perciben los diferenciales niveles de exigencia a los que se exponen los estudiantes durante la enseñanza media, desconfiando de las políticas evaluativas que muchas veces exhiben calificaciones infladas que no son el real reflejo del aprovechamiento educativo. Estas instituciones valorarán el factor PSU por sobre el de Trayectoria Escolar, más allá de la constatación que hagan respecto de la correlación que tengan las características sociofamiliares con determinados niveles de logro en las pruebas estandarizadas.

Por otra parte, existiría un conjunto de instituciones que valorarían más significativamente la Trayectoria Escolar en la confianza de que las calificaciones y la posición relativa del alumno respecto de sus pares expresarían un conjunto de habilidades y aptitudes desarrolladas en los 4 años previos de enseñanza que los prepararía adecuadamente para la vida universitaria, controlando en parte el sesgo sociocultural que tendrían las pruebas de admisión, las cuales resultan altamente discriminatorias cuando adquieren un rol hegemónico como factor de ponderación. A favor de esta postura institucional se encuentran dos hechos esenciales: primero, los fines de la enseñanza media, al que adhieren explícita o implícitamente tanto la demanda como la oferta educativa, se encuentran fuertemente orientados hacia la continuación de estudios superiores, donde la enseñanza secundaria es la fase previa encargada de preparar para el desarrollo de tal trayectoria. En segundo lugar, existe una vinculación entre los contenidos mínimos curriculares obligatorios impartidos por los diferentes establecimientos de educación media y las pruebas de selección universitaria. En consecuencia, estos test de admisión pretenden medir el nivel de conocimiento o dominio logrado por el estudiante sobre iguales contenidos ya evaluados por el establecimiento, con el único atributo de hacerlo en forma independiente de este. Sería, por tanto, un contrasentido asignar excesiva ponderación al componente PSU en detrimento del reconocimiento a la Trayectoria Escolar, máxime cuando la evidencia ha revelado incongruencias y dificultades para construirlo como un predictor igualitario de rendimiento académico universitario. Vinculado a esto se encuentra la desventaja de los estudiantes que no tienen la posibilidad de una preparación pre-universitaria para rendir la PSU, la que además se vale de contenidos que menos del 50\% de los establecimientos municipales dicta completamente (Ortiz, Parra y Pérez, 2011). Por lo demás, un alumno de buen rendimiento académico se puede adaptar a las exigencias de diferentes contextos educativos y actualizar plenamente sus potencialidades intelectuales independiente de su medio social, familiar y educacional de origen, ya que siente placer por aprender y posee un locus de control interno que lo hace superar las dificultades que se le presentan (Gil, 2006).

Post ajuste del ranking según autonomía administrativa en 2014, el sistema de ingreso a la educación superior ha experimentado cambios que buscan interrumpir la reproductibilidad de las lógicas estructurantes del sistema. Los nuevos lineamientos frente a la Trayectoria 
Escolar demandan un posicionamiento institucional respecto a la eventual flexibilización de los requerimientos que históricamente han servido de criba. La vinculación tradicionalista —elitista - entre selectividad y excelencia expresada mediante la excelsa valoración al conocimiento adquirido en circunstancias de competencia desigual, no únicamente relega el rendimiento académico previo a la bondadosa meritocracia institucional, sino que además se vuelve un componente ideológico que limita las posibilidades de cambio del sistema. A esto se suma que los procesos de selección son instancias únicas y acotadas (una vez al año) donde se tiende a la superposición de resultados sobre procesos (4 a 5 años), realidad que involucra un peso mayor para la preparación preuniversitaria ad hoc en comparación a la regular de 4 años.

Cuadro 3. Valoración de la Trayectoria Escolar asignada por los programas ofertados según nivel de selectividad en el proceso de selección 2014

\begin{tabular}{|l|c|c|c|c|}
\hline Nivel de selectividad & $20 \%$ & $25 \%-30 \%$ & $35 \%-40 \%$ & $>40 \%$ \\
\hline Alta & 7,9 & 16,7 & 62,3 & 13,2 \\
\hline Media & 19,3 & 21,1 & 31,9 & 27,7 \\
\hline Baja & 5,9 & 19,8 & 28,5 & 45,9 \\
\hline Total & 11,6 & 20,1 & 32,6 & 35,7 \\
\hline
\end{tabular}

En este nuevo escenario de ajuste de los factores de ponderación, una vez rendida la batería de pruebas que contempla el proceso de selección, queda la expectación de los resultados y con base en la definición de las posibilidades de quedar seleccionado en alguna opción de predilección del postulante. Expectación que si bien continúa significando un espacio de incertidumbre, es menos relevante que antaño, toda vez que aproximadamente 2 de cada 5 puntos ya han sido determinados por la valoración de la Trayectoria Escolar. Y si bien existe una dispersión en los niveles de valoración, la gran mayoría de los programas $(68,3 \%)$ asigna a este componente valoraciones por sobre los 30 puntos ponderados (ver Cuadro 3).

No obstante ello, cabe hacer presente que la relevancia que adquiere el desempeño escolar previo, vía Ranking o NEM, diferirá de acuerdo al nivel de selectividad del programa, tal y como se puede observar en el Cuadro 3. Opciones de valoración que fluctuarían según la proporción de la demanda histórica ubicada en determinado rango de puntaje PSU.

Como se mencionó anteriormente, una de las posturas institucionales frente a los nuevos procesos de selección sería mantener ponderaciones altas al componente PSU, opción preferencial de los programas más selectivos. La oferta educativa fuertemente selectiva, definida por aquel programa donde al menos el 50\% de su matrícula histórica registraba puntajes PSU (Lenguaje y Matemáticas) superiores a los 650 puntos, se caracteriza actualmente por asignar menos relevancia a la Trayectoria Escolar. Tan solo un 13,2\% de estos programas selectivos le asigna una ponderación superior al $40 \%$. Como contraparte, el $45,9 \%$ de los programas de baja selectividad otorga una valoración a la trayectoria escolar 
superior a los 40 puntos ponderados. En la medida que los programas de baja selectividad se caracterizan por tener alumnos de bajo rendimiento PSU, por cuanto no más del $50 \%$ de su matrícula histórica de referencia supera los 550 puntos PSU, es lógicamente natural visualizar una depreciación de los componentes tradicionales de selección.

Las políticas de asignación de valores a los factores de ponderación en este último segmento se pueden considerar inclusivas desde la perspectiva y objetivos que impulsaron la reforma al sistema de selección. Al valorar NEM y Ranking cerca del máximo permitido como dimensión, cerca de 1 de cada 2 programas $(45,9 \%)$ no solo expande las posibilidades de ingreso a la educación superior de cierto tipo de estudiantes, sino que además cambia el enfoque de la selectividad a procesos y resultados por igual. Ratifica, de este modo, la importancia de la formación escolar anterior optando por conseguir resultados y fines similares a los históricos a través de medios distintos. Cabría preguntarse si esta resignificación de la Trayectoria Escolar se traducirá en una relativa modificación del perfil de ingreso del estudiante, más allá de un artificioso aumento de los puntajes de corte, toda vez que los datos informan que esta tipología de estudiantes tiende a exhibir significativos mejores puntajes en la Trayectoria Escolar que los alcanzados en las pruebas de admisión.

Cuadro 4. Valoración de la Trayectoria Escolar en asignada por las carreras de Medicina, Pedagogía y Trabajo Social en los procesos de selección 2012, 2013 y 2014

\begin{tabular}{|l|c|c|c|c|c|c|c|c|}
\hline \multirow{2}{*}{ Carreras } & \multicolumn{2}{|c|}{$20 \%$} & \multicolumn{2}{c|}{$25 \%-30 \%$} & \multicolumn{2}{c|}{$35 \%-40 \%$} & \multicolumn{2}{c|}{$>40 \%$} \\
\cline { 2 - 9 } & 2013 & 2014 & 2013 & 2014 & 2013 & 2014 & 2013 & 2014 \\
\hline Medicina & 18,2 & 8,7 & 54,5 & 13,0 & 22,7 & 47,8 & 4,5 & 30,4 \\
\hline Pedagogía & 8,6 & 7,0 & 47,6 & 20,0 & 24,3 & 30,5 & 19,5 & 42,5 \\
\hline Trabajo Social & 13,6 & - & 18,2 & 8,7 & 50 & 30,4 & 18,2 & 60,9 \\
\hline Total & 10 & 6,5 & 45,4 & 18,3 & 26,6 & 32,1 & 17,6 & 43,1 \\
\hline
\end{tabular}

A fin de ilustrar de mejor manera las estrategias institucionales de valoración de la Trayectoria Escolar, el Cuadro 4 incluye casos concretos de programas pertenecientes a los diferentes niveles de selectividad. En general, en lo que viene a ser una constante observada, se aprecia una disminución de los componentes tradicionales de selección. No obstante ello, con base en la autonomía administrativa exhibida por las instituciones, el aumento de las ponderaciones a la Trayectoria Escolar registra variaciones considerables en cada uno de los tres programas analizados. En los programas de Medicina, carreras altamente selectivas, con prestigio social y opción educativa característica de los estratos sociales altos, se observa una tendencia a asignar mayor ponderación a los factores tradicionales de selección, donde tan solo el 30,4\% de sus programas optan por asignar una valoración superior al $40 \%$ a la Trayectoria Escolar. Cifras que distan significativamente en magnitud del comportamiento registrado por carreras como Trabajo Social a igual respecto, donde el $60,9 \%$ de sus programas asigna sobre 40 puntos porcentuales a la Trayectoria Escolar. Son precisamente las carreras menos selectivas y que se caracterizan por ser opción educativa de 
sectores socioeconómicos más vulnerables, muchos de los cuales son primera generación en acceder a la educación superior, los que tienden a otorgarle mayor valoración a este componente.

En síntesis, con independencia del nivel de selectividad, tipo de institución o carrera que se trate, los fines corporativos detrás del proyecto de la educación superior no pueden ser abandonados, de modo que las estrategias de asignación de puntaje, pudiendo ser bien intencionadas, no son ingenuas ni neutrales a los intereses institucionales. La predilección a la hora de valorar la Trayectoria Escolar que hacen los programas educativos es tan solo una pieza del panorama general, que en concordancia a los supuestos mencionados anteriormente, forma parte de elaboradas estrategias de reclutamiento que dan cuenta de una adaptabilidad más o menos forzada a este nuevo contexto de competencia entre instituciones. Queda por determinar en qué medida estas estrategias de adhesión al nuevo sistema de selección logran constituirse en un real factor de inclusión que modifique el perfil de admisión, más allá de una simple y artificiosa elevación de los por sí ya reducidos puntajes que evidencian programas de baja selectividad.

\section{CONCLUSIONES}

La reforma del sistema de admisión universitaria en Chile y la incorporación del Ranking como su componente esencial en la búsqueda de hacer más inclusivo el acceso a la educación superior, implicaría, por parte de las instituciones, reestructurar las políticas de reclutamiento de sus programas de formación superior en cuanto a lo que exigen para formar parte de su comunidad.

La necesaria redistribución de las ponderaciones otorgadas a los factores de selección universitaria a causa de la inclusión de Ranking de Notas desencadena una serie de respuestas deliberadas por parte de las universidades vinculadas al Sistema Único de Admisión del CRUCH. La nueva perspectiva que abre la mayor valoración de la Trayectoria Escolar (NEM y Ranking) en lo que tradicionalmente se consideraba como necesario para ingresar a la educación superior, constituye el eje temático medular en este trabajo.

Sujeto a los bemoles que presenta este nuevo escenario, el análisis de las diferentes ponderaciones otorgadas a los factores de selección 2014, divididos en dimensión PSU y Trayectoria Escolar, muestran que la batería de pruebas estandarizadas, que ha servido como indicador tradicional para la selección de estudiantes aptos para sobrellevar la exigencia de la educación superior, ha visto mermada su relevancia y hegemonía. Esto en la medida, como es lógico, del aumento de la incidencia o preponderancia del componente Trayectoria Escolar dentro del puntaje ponderado. Situación más o menos generalizada, no obstante las diferentes valoraciones a partir de las características particulares que posee cada carrera o institución universitaria.

La modalidad e intensidad diferenciada con la que las instituciones incorporan los componentes de Trayectoria Escolar, y de manera especial el Ranking, configura en lo inmediato una brecha virtual entre instituciones selectivas o no selectivas de naturaleza pública o privada que reproduciría las mismas lógicas de segmentación existentes en la enseñanza media.

Al agrupar los programas según su nivel de selectividad, independiente del tipo de institución que los oferte, se observan magnitudes diferenciadas en la valoración de la 
Trayectoria Escolar, siendo las más selectivas quienes menor ponderación le asignan. Lo anterior evidencia que los fines que persigue uno y otro programa son diferentes.

Algunos toman posiciones más osadas o "arriesgadas" reduciendo significativamente la ponderación al factor PSU para favorecer la Trayectoria Escolar, basados en el supuesto que la fuerte adhesión a esta medida de inclusión no implica necesariamente una pérdida de calidad de los estudiantes que ingresan a sus programas. Otros, en cambio, toman posiciones más cautelosas moviéndose en un rango de confianza cercano a los mínimos establecidos por ley, siempre y cuando esto no implique un deterioro en la preponderancia del rendimiento obtenido por el postulante en la dimensión PSU. Factor al cual ya le reconocen capacidad predictiva en concomitancia con sus fines.

El nivel de adhesión al Ranking se constituye en una medida de resistencia o adaptación a los cambios del mecanismo de selección. Esto se operacionaliza en estrategias de reclutamiento con diferentes gradientes entre selectividad e inclusión existiendo, por un lado, las que se pueden definir como tradicionales que otorgan una alta valoración a la PSU como reflejo de las capacidades de los estudiantes, y, por otro, las flexibles que reconocen las habilidades desarrolladas en los procesos educativos anteriores expresados en la Trayectoria Escolar. En consecuencia y estrechamente vinculado a lo anterior, se puede establecer que el acceso a los programas no está configurado exclusivamente por el rendimiento obtenido en cada uno de los factores de ponderación (PSU y Trayectoria Escolar) por parte del estudiante, sino que también en las valoraciones que las instituciones educativas hacen de ellos, pudiéndose afirmar que, finalmente, son las universidades las que eligen, y sus políticas las barreras a superar para ingresar a la educación superior. Finalmente, es aquí donde se define el nivel de logro de los objetivos de inclusión al sistema, relacionado con reales posibilidades de acceso y permanencia en la educación superior.

\section{REFERENCIAS BIBLIOGRÁFICAS}

Bellei, C. (2013). El estudio de la segregación socioeconómica y académica de la educación chilena. Estudios Pedagógicos, 39, 325-345.

Chiroleu, A. (2009). La inclusión en la educación superior como política pública: tres experiencias en América Latina. Revista Iberoamericana de Educación, 48(5), 1-15.

Cliffordson, C., \& Askling, B. (2006). Different Grounds for Admission: Its effects on recruitment and achievement in medical education. Scandinavian Journal of Educational Research, 50, 45-62.

Contreras, D., Gallegos, S., \& Meneses, F. (2009). Determinantes del desempeño universitario: ¿Importa la habilidad relativa? Revista Calidad en la Educación, 39, 17-48.

Contreras, M., Corbalán, F., \& Redondo, J. (2007). Cuando la suerte está echada: Estudio cuantitativo de los factores asociados al rendimiento en la PSU. Revista Electrónica Iberoamericana sobre Calidad, Eficacia y Cambio en Educación, 5, 259-263.

Demre. (2014). Bases de datos oficiales resultados procesos de admisión a las universidades chilenas del Departamento de Evaluación, Medición y Registro, Universidad de Chile. Santiago: autor

Díaz, E., Himmel, E., \& Maltes, S. (1990). Evolución histórica del sistema de selección a las Universidades Chilenas, 1967-1989. En M. J. Lemaitre, La educación superior en Chile: un sistema de transición. Santiago de Chile: Corporación de Promoción Universitaria (CPU), Colección Foro de la Educación Superior.

Espinoza, O. (2015). Equidad e inclusión en el sistema de educación superior de Chile. En O. Espinoza (Ed.), Equidad e inclusividad en la educación superior en los países andinos: los 
casos de Bolivia, Chile, Colombia y Perú (pp. 93-133). Chile: Ediciones Universidad UCINF.

Gallegos, S., \& Meneses, F. (2007). ¿Es eficiente el sistema de ingreso a la universidad? El uso del ranking en la Universidad Católica de Chile. Santiago de Chile: Universidad Católica de Chile.

Geiser, S., \& Studley, R. (2002). UC and the SAT: Predictive validity and differential impact of the SAT I and SAT II at the University of California. Educational Assessment, 8(1), 1-26.

Gil, F. (2006). Acceso a las universidades: una propuesta. Santiago de Chile: Cuadernos del foro nacional de educación de calidad para todos.

Gil, F., \& Canto, C. (2012). The case of the propedeutico program at Universidad de Santiago de Chile (USACh). Pensamiento educativo, 49(2), 65-83.

Gil, F., Paredes, R., \& Sánchez, I. (2013). El ranking de las notas: inclusión con excelencia. Centro de políticas públicas de la UC, 8(60), 1-19.

Larroucau, T. (2014). Ranking de Notas Proceso de Admisión 2014. Santiago: CRUCH.

Larroucau, T., Ríos, I., \& Mizala, A. (2013). Efectos de la incorporación del ranking de notas en la selección universitaria. Santiago: DEMRE.

Medina, A., Aguirre, M., \& Luengo, L. (2014). Predictibilidad de las notas de enseñanza media según establecimiento de origen sobre el rendimiento académico en estudiantes de Odontología. Educación Médica Superior, 28(1), 65-73.

Meneses, F., \& Blanco, C. (2006). La posición en el Ranking y el puntaje de ingreso en la PUC: implicancias prácticas. Santiago: Departamento de Estudio MINEDUC.

Meneses, F., \& Toro, J. (2012). Predicción de notas en derecho de la Universidad de Chile: ¿sirve el ranking? ISEES, 10, 43-58.

Meneses, F., Parra, A., \& Zenteno, L. (2005). ¿Se Puede Mejorar el Sistema de Ingreso a las Universidades Chilenas? El uso del ranking en la Universidad Católica de Chile, Universidad de Chile y Universidad de Santiago de Chile. Santiago: Universidad de Chile.

Ortiz, L., Parra, P., \& Pérez, C. (2011). Prueba de Selección Universitaria, rendimiento en enseñanza media y variables cognitivo-actitudinales en alumnos de Medicina. Revista de Educación, Ciencia y Salud, 8(2), 120-127.

Román, C. (2012). ¿Qué hay detrás del buen rendimiento escolar en los estudiantes que ingresan a la universidad a través del programa propedéutico de la UCSH (Chile). Investigación arbitrada, 16(55), 331-338.

Sverdlick, I., Ferrari, P., \& Jaimovich, A. (2005). Desigualdad e inclusión en la educación superior. Un estudio comparado en cinco países de América Latina. Buenos Aires: Fundación Laboratorio de Políticas públicas.

Valdivieso, P., Antivilo, A., \& Barrios, J. (2006). Caracterización educacional y sociodemográfica de los estudiantes que rinden la PSU, postulan y se matriculan en las universidades reunidas en el consejo de rectores. Calidad en la educación, 24, 319-361. 